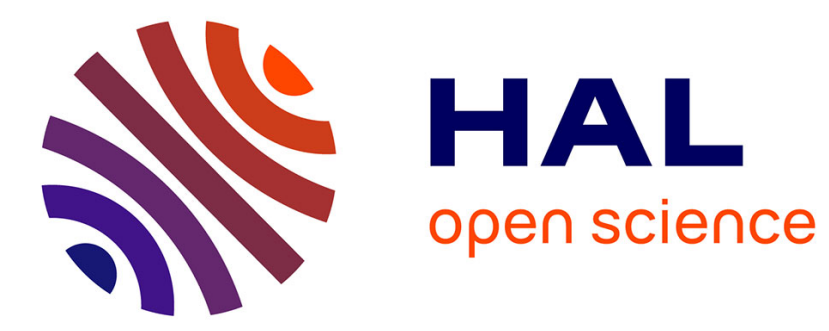

\title{
Crystallization of non-Brownian Spheres under Horizontal Shaking
}

Olivier Pouliquen, Maxime Nicolas, P. D. Weidman

\section{To cite this version:}

Olivier Pouliquen, Maxime Nicolas, P. D. Weidman. Crystallization of non-Brownian Spheres under Horizontal Shaking. Physical Review Letters, 1997, 79, pp.3640 - 3643. 10.1103/PhysRevLett.79.3640 . hal-01440072

\section{HAL Id: hal-01440072 https://hal.science/hal-01440072}

Submitted on 18 Jan 2017

HAL is a multi-disciplinary open access archive for the deposit and dissemination of scientific research documents, whether they are published or not. The documents may come from teaching and research institutions in France or abroad, or from public or private research centers.
L'archive ouverte pluridisciplinaire HAL, est destinée au dépôt et à la diffusion de documents scientifiques de niveau recherche, publiés ou non, émanant des établissements d'enseignement et de recherche français ou étrangers, des laboratoires publics ou privés. 


\title{
Crystallization of non-Brownian Spheres under Horizontal Shaking
}

\author{
O. Pouliquen, M. Nicolas, and P.D. Weidman* \\ LadHyX, CNRS UMR 156, École Polytechnique, 91128 Palaiseau Cedex, France
}

(Received 31 July 1997)

\begin{abstract}
Until now, it has been observed that a collective handling of uniform spheres could only lead to a random close packing of 0.64 maximum volume fraction. In this Letter, we show that denser crystalline arrangements can be obtained when beads are poured at low flow rates into a horizontally shaken container. A parallel is suggested between this process and that of colloidal sedimentation which also yields crystalline structure. [S0031-9007(97)04421-9]
\end{abstract}

PACS numbers: 46.10.+z, 61.50.-f, 81.20.Ev, 83.70.Fn

Packings of uniform spheres have been extensively studied both by the powder technology community [1,2] and by physicists who consider hard spheres systems as a model for simple liquids [3]. It is well known (although not rigorously demonstrated $[4,5]$ ) that the volume fraction of the packing, defined as the ratio of the volume occupied by the spheres to the total volume, cannot exceed 0.74 , corresponding to the regular crystal arrangements (for example, hexagonal or face-centered cubic). However, spheres simply released in a box do not spontaneously arrange into a crystalline formation. Even when vibrations are imposed to compact the arrangement, the maximum volume fraction obtained is 0.64 (in the limit of large boxes) [6-9]. This is called the random close packing limit and is commonly considered as the maximum density that can be reached by collectively handling the particles as reported by an anonymous author [10]: "ball bearings and similar objects have been shaken, settled in oil, stuck with paint, kneaded inside rubber balloons and all with no better result than a density of about 0.636 ."

Although this limit seems to be well defined, some studies in the literature report that higher densities corresponding to regular arrangements can be obtained. Scott et al. observed in a short note [11] that cyclic shear motion can induce packing with a density higher than 0.64. Owe Berg et al. [12] carried out experiments on the packing of spheres obtained under different shaking processes and concluded that "three dimensional shaking" (shaking with an horizontal component) can lead to a crystallization. More recently, Vanel et al. [13] obtained a regular packing of steel beads by vertically vibrating the container. However, all these experiments deal with a very small number of particles and the thickness of the crystal does not exceed 20 particles. Under such conditions, the packings are certainly dramatically influenced by the boundaries. To our knowledge, no process has been reported for creating large crystals of non-Brownian spheres.

On the other hand, in the case of Brownian hard spheres sensitive to thermal fluctuations, spontaneous crystallization can occur [14-17]. The slow sedimentation of colloidal spherical particles in a fluid with the same index of refraction (in order to minimize the van der Waals forces) yields a dense crystalline sediment of density around 0.67 [15]. The crystallization is in this case controlled by the temperature: as a result of Brownian motion, the spheres reaching the free surface of the sediment rearrange spontaneously into an equilibrium ordered state.

In this paper we present a method inspired by the colloidal crystallization for creating crystalline arrangements of non-Brownian spheres. The method consists in continuously pouring the spheres into a box subjected to horizontal vibrations. The vibrations allow the particles to rearrange and play a role similar to the temperature in the colloidal sedimentation. Whereas many reports have been devoted to the dynamics of a granular media $[18,19]$ and its compaction $[1,20,21]$ under vertical vibrations, few have been concerned with horizontal vibrations [22,23] and to our knowledge no crystallization results have been reported.

The experimental setup is presented in Fig. 1. A glass box $12 \mathrm{~cm} \times 12 \mathrm{~cm} \times 25 \mathrm{~cm}$ is fixed on a table which can horizontally move on linear bearings. A

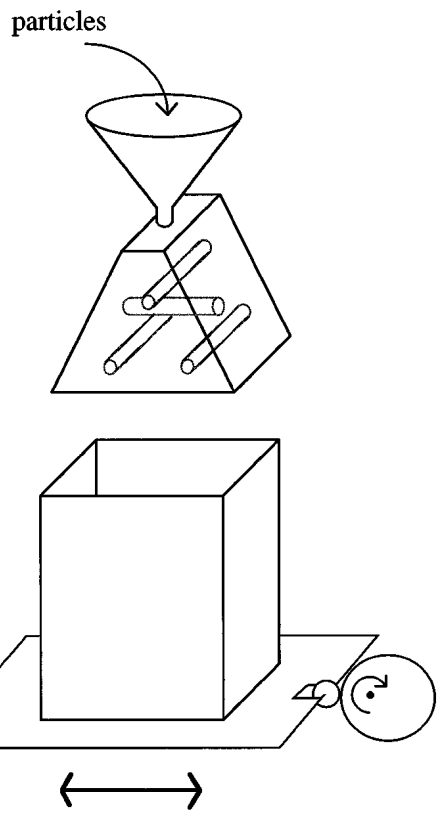

FIG. 1. Experimental setup. 
quasisinusoidal motion $A \sin (\omega t)$ is induced to the table by the mean of an eccentric wheel driven by a motor. The particles fall from a glass funnel into the box through a set of horizontal cylindrical bars: the chaotic motion of the particles induced by the multiple collisions with the bars provides a uniform distribution of the particles over the surface of the box. The particle flow rate can be varied by using funnels having different outlet diameters. The particles we used were spherical glass beads $1.98 \pm 0.06 \mathrm{~mm}$ in diameter, with a density $\rho=$ $2.64 \mathrm{~g} / \mathrm{cm}^{3}$. Many parameters can influence the granular dynamics, but in this paper we focus on the three major control parameters:

(i) The relative acceleration of the vibration $\Gamma=$ $A \omega^{2} / g$ where $A$ and $\omega$ are the amplitude and the pulsation of the vibration, and $g$ the gravitational acceleration.

(ii) The filling rate $Q$, number of particles falling in the box per second per unit surface area [in (particle $\left./ \mathrm{s}) / \mathrm{cm}^{2}\right]$.

(iii) The relative amplitude of vibration $A / d$, where $d$ is the particle diameter.

The influence of other important effects like the surface properties of the particles, the density of the beads, and the falling height of the particles has not yet been studied systematically.

To characterize the crystallization, we computed the final volume fraction $\phi$ of the packing simply by measuring the height and the weight of the final packing. This method takes into account the boundary effects and thus gives densities lower than in the infinite container limit [6].

Before each experiment, the walls of the box are cleaned with an antistatic agent in order to minimize the electrostatic interaction. Horizontal vibration is then imposed to the empty box before the particles are poured into the funnel. The experiment is stopped when the height of the packing reaches at least $20 \mathrm{~cm}$ ( $\sim 100$ particle diameters). When the particle flow rate is low and the relative acceleration is sufficiently strong, a very dense and ordered packing is obtained. Figure 2 shows the free surface of such a packing. We have

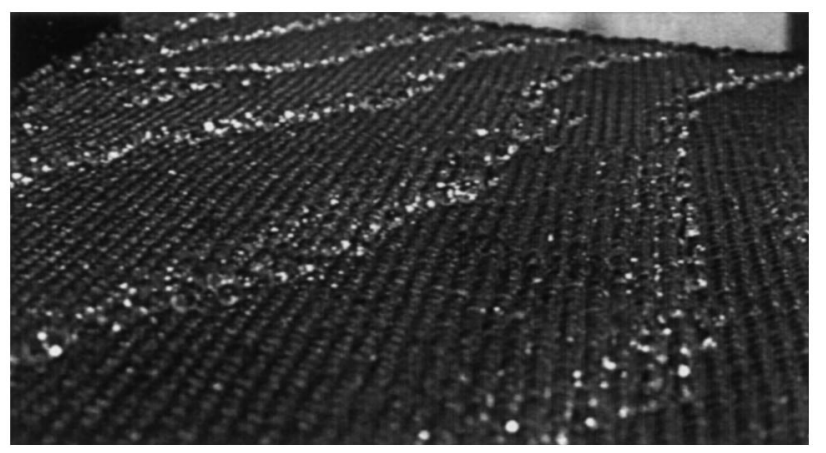

FIG. 2. The free surface of a packing obtained for $\Gamma=1$, $Q=2($ particle $/ \mathrm{s}) / \mathrm{cm}^{2}, A / d=1.1$. In order to enhance the crystal-like structure, we have artificially created steps on the free surface by slightly inclining the box from horizontal. verified that the organized structure observed at the free surface in Fig. 2 is the signature of an internal organized structure by using the elegant method proposed by Berg et al. [12]: at the end of the experiment, water is poured into the container and frozen. The ice block then can be carefully cut apart to reveal the internal packing structure. As in colloidal crystallization, the packings we observed are regular but consist of a superposition of randomly stacked hexagonal layers perpendicular to gravity [24]. We return to a discussion of this point later in the paper.

Careful observation of the system during the filling reveals that the crystallization process is entirely controlled by the dynamics of the free surface. The vibrations serve to fluidize the uppermost layers of the packing (between 0 and 6 layers, depending on the acceleration) [23], while the rest of the particles remain fixed in the frame of the accelerating box. In this fluidized layer, the particles experience both horizontal random motion and shearing. The arrangement taken by the particles seems to result from the combination of these two factors. The active zone being restricted to the free surface, the only method for creating a large crystalline arrangement is to continuously supply new particles to the free surface. A horizontal shaking applied to a randomly filled box leads to a thin layer of crystal phase at the free surface, but the rest of the material below remains in the initial random state. It should be noted that convective particle motion was not observed at the low values of $\Gamma$ covered in the present experiments.

The two necessary ingredients for creating a crystal are thus an acceleration sufficiently large to fluidize the free surface, and a moderately low flow rate to allow time for the free surface particles to organize in a regular pattern before being covered by the new particles showered from above. We have systematically studied the influence of the acceleration, $\Gamma$, and of the flow rate, $Q$, by measuring the final volume fraction of the packings. The results are presented in Fig. 3. For low accelerations, $\Gamma \lesssim 0.4$, the packings are random with a density 0.625 , independent of the filling rate. This value is compatible with the random close packing density measured by Scott [6] in containers having the same relative size (box length/particle diameter) as in our experiment. For higher acceleration, crystallization is observed but strongly depends on the filling rate. For very low filling rates $\left[Q \lesssim 2(\right.$ particle $\left./ \mathrm{s}) / \mathrm{cm}^{2}\right]$ the final volume fraction $\phi$ is equal to 0.67 , the maximum we were able to obtain. This value is independent of $\Gamma$ in the range allowed with our experimental setup. For intermediate flow rates, $2 \lesssim$ $Q \lesssim 120($ particle $/ \mathrm{s}) / \mathrm{cm}^{2}$, the volume fraction decreases with increasing $Q$ [Fig. 3(b)] at constant acceleration. This tendency is a direct consequence of the creation of more and more defects in the crystal. In this range of flow rate, a decrease of the volume fraction is also observed when the relative acceleration rises above one, in which case the fluctuating motion induced by the horizontal 

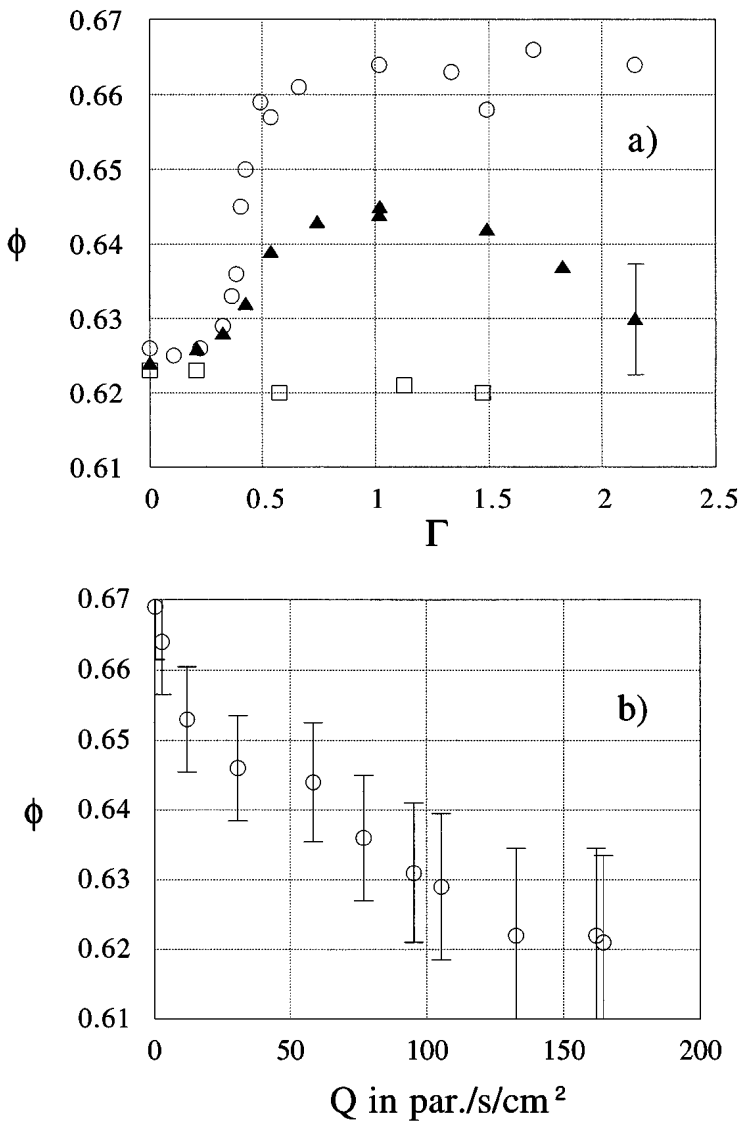

FIG. 3. (a) Final volume fraction as a function of the relative acceleration $\Gamma$ for $A / d=1.1$ and for different filling rates. ॰: $Q=2.8$ (particle/s) $/ \mathrm{cm}^{2} ; \mathbf{\Lambda}: Q=60 ; \square: Q=120$. (b) Final volume fraction as a function of the filling rate for $\Gamma=1$ and $A / d=1.1$.

vibration becomes increasingly important. Finally, for high filling rates $Q \gtrsim 120$ (particle $/ \mathrm{s}) / \mathrm{cm}^{2}$ crystallization is no longer observed.

We have also investigated the influence of the dimensionless vibration amplitude $A / d$ varied over the range $0.35-10$ at $\Gamma=0.66$ and a flow rate $Q=12($ particle $/ \mathrm{s}) / \mathrm{cm}^{2}$. In this range the final density does not depend on $A / d$ as long as $A / d>1$, the density being $\phi=0.655$. For vibration amplitudes less than the particle diameter, $A / d<1$, a slight decrease in the compacity seems to appear, the final value $\phi=0.645$ attained at $A / d=0.35$.

The experiments presented here were carried out in a square section box with flat walls. One can legitimately wonder to what extent the crystallization is initiated by the flat boundary conditions. We thus performed a control experiment using irregular boundaries made of glass beads $4 \mathrm{~mm}$ in diameter randomly glued to the bottom surface and on the four lateral walls of the glass box. The volume fraction of the random packing obtained when the beads are poured in this roughened box at $Q=3($ particle $/ \mathrm{s}) / \mathrm{cm}^{2}$ with no external vibration is $\phi=0.615$. When a horizontal acceleration $\Gamma=1$ is imposed, the final density is $\phi=0.645$. The study of the internal structure of the packing exhibits a crystallike structure everywhere except in a layer of 8 particle diameters adjacent to the walls, showing that sphere crystallization is an intrinsic behavior of the system, and not the result of flat boundaries. We conclude that ordered nature of the packings is insensitive to wall roughness conditions.

However, some specific boundaries can dramatically influence the precise structure of a crystalline arrangement. For the flat or irregular boundaries considered above, the crystalline packings we have created are formed by the superposition of hexagonal-patterned layers [Fig. 4(a)]. However, there exist two ways of forming a new hexagonal layer on top of a previous one. The final arrangement is thus, strictly speaking, not a crystal, but a sequence of randomly stacked hexagonal packings perpendicular to gravity. This vertical, one-dimensional disorder is well known in colloidal sedimentation [24]. The crystalline sediment is not a perfect crystal, and this leads to some difficulties for technological applications. Recently, van Blaaderen et al. [17] have proposed a method for controlling the crystal orientation and producing large single colloidal crystals. Their idea is to create the sediment starting with a square-patterned substrate. When the mesh of the substrate is exactly equal to the particle diameter, the square pattern corresponds to the (100) plane of a face-centered-cubic (fcc) packing. Since there is only one way for the beads to arrange themselves on the square pattern, a perfect fcc crystal can be formed. We have applied the same method in our system of non-Brownian spheres, by placing a square wire netting on the bottom surface before filling the box under horizontal vibrations. The mesh was $2 \mathrm{~mm}$ and oriented parallel to the vibration direction (otherwise the square pattern is lost after a few

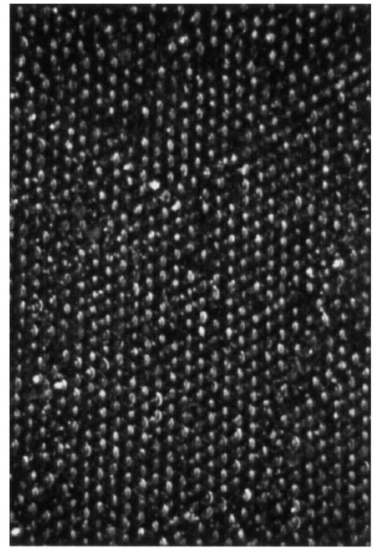

a)

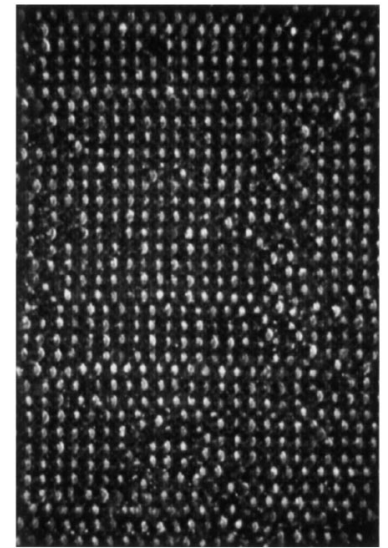

b)
FIG. 4. The free surface structure of packings 40 layers thick obtained in a box with: (a) flat bottom wall; (b) squarepatterned bottom wall. 
layers). Under such conditions, we obtained a fcc crystal exhibiting a square pattern at the free surface [Fig. 4(b)]. The efficiency of the method seems to be only limited by boundary effects: some zones of hexagonal packing nucleate close to the walls parallel to the direction of oscillation. Their size grows as the height of the packing increases, invading finally the entire box. The maximum height of the fcc crystal that can be created (50 particle diameters in our box) is thus controlled by the width of the container.

In conclusion, we have shown that crystalline packings denser than random close packing can be obtained by collectively handling the particles. The method of filling under horizontal vibration presents strong analogies with colloidal crystallization. However, the particle dynamics are different, since horizontal vibration under a vertical gravitational field is not a priori equivalent to thermal fluctuations. Further investigations are thus necessary in order to precisely understand how the horizontal vibrations lead to the spontaneous arrangement of quasicrystalline particle structures.

This work has benefited from stimulating discussions with P. Gondret and R. Monasson. We thank A. Garcia, T. Lescuyer, and J. Webert for technical assistance and R. T. Goodwin for a critical reading of the manuscript.

*Permanent address: Department of Mechanical Engineering, University of Colorado, Boulder, CO 80309.

[1] H. H. Hausner, K. H. Roll, and P. K. Johnson, Vibratory Compacting, Principles and Methods Vol. 2 (Plenum Press, New York, 1967).

[2] D. J. Cumberland and R. J. Crawford, Packing of Particles, Handbook of Powder Technology Vol. 6 (Elsevier, Amsterdam, 1987).
[3] J. D. Bernal, Proc. R. Soc. London A 280, 299 (1964).

[4] N. J. A. Sloane, Sci. Am. 250, 92 (1984).

[5] J. H. Conway and N. J. A. Sloane, Sphere Packing, Lattices and Groups, Series of Comprehensible Studies in Mathematics Vol. 290 (Springer, Berlin, 1988).

[6] D. G. Scott, Nature (London) 188, 908 (1960).

[7] M. A. Slifkin, Nature (London) 193, 465 (1962).

[8] H. Susskind and W. Becker, Nature (London) 212, 1564 (1966).

[9] J. G. Berryman, Phys. Rev. A 27, 1053 (1983).

[10] Anonymous, Nature (London) 239, 488 (1972).

[11] D. G. Scott, J. Chem. Phys. 40, 611 (1964).

[12] T. G. Owe Berg, R. L. McDonald, and R. J. Trainor, Jr., Powder Technol. 3, 183 (1969).

[13] L. Vanel, A. D. Rosato, and R. N. Dave, Phys. Rev. Lett. 78, 1255 (1997).

[14] P. N. Pusey and W. van Megen, Nature (London) 320, 340 (1986).

[15] K.E. Davis, W. B. Russel, and W. J. Glantschnig, Science 245, 507 (1989).

[16] P. N. Pusey et al., Phys. Rev. Lett. 63, 2753 (1989).

[17] A. van Blaaderen, R. Ruel, and P. Wiltzius, Nature (London) 385, 321 (1997).

[18] S. Douady, S. Fauve, and C. Laroche, Europhys. Lett. 8, 621 (1989).

[19] F. Melo, P. B. Umbanhowar, and H. L. Swinney, Phys. Rev. Lett. 75, 3838 (1995).

[20] J. E. Ayer and F. E. Soppet, J. Am. Ceram. Soc. 48, 180 (1965).

[21] J. B. Knight, et al., Phys. Rev. E 51, 3957 (1995).

[22] P. Evesque, P. Alfonsi, C. Stefani, and B. Barbe, C. R. Acad. Sci. Paris Serie II 311, 393 (1990).

[23] S. G. K. Tennakoon and R.P. Behringer (to be published).

[24] M. S. Elliot, B. T.F. Bristol, and W.C.K. Poon, Physica (Amsterdam) 235A, 216 (1997). 